\title{
Merit and important point of Near Infrared (NIR) Thermography
}

by H. Uemura

\author{
FLIR Systems Japan K.K., Kami-Osaki 2-13-17, Shinagawa-ku, 141-0021, Tokyo, Japan,
} hideyuki.uemura@flir.jp

\section{Abstract}

Thermography is recognized generally that Mid-Wave Infrared (MWIR : 3-5um) or Long-Wave Infrared (LWIR : 7-14um) wave length is used. It is because on its wavelength the electromagnetic wave radiates enough to be detected at room temperature on the detector of thermography camera. Near Infrared (NIR : 0.9-1.7um) can also be used as thermography. On this paper, we introduce NIR thermography, especially the merit, important and noticed point, or limitation. Also introduce some application examples with some images.

\section{Introduction}

Thermography camera technology is evolving drastically recently on both of cooled and uncooled cameras. Uncooled camera price has got much cheaper and much smaller. Also cooled camera has got much faster and smaller. Thermography camera technology mainly depends on the detector one, and still manufacturer is developing for cheaper, faster cameras.

Customers can buy thermography with the cheaper budget, however, there is some limitation to use it by some physics reasons. Mid-Wave (MWIR : 3-5um) or Long-Wave (LWIR : 7-14um) infrared ray is opaque by the glass, therefore optics has to be used by germanium or silicon. It makes the optics to be expensive. As for the same reason, MWIR/LWIR thermography cannot see anything, cannot measure the temperature of the material through glass. In the area of NIR, optics are made by glass, and NIR thermography can measure the temperature through glass. Besides, there are some more merit of NIR thermography.

\section{Near Infrared camera}

Historically, Near Infrared camera is used for the long time. In the nighttime and no visible light, NIR camera can see the target by using NIR floodlight. Or for confirmation of the exact person at the automatic teller machine of the bank, NIR camera can detect the vein on the finger or palm. For this kind of applications, CCD made by silicon is used and the wavelength is around 1 um.

For longer than 1um, MCT (Mercury Cadmium Tellurium) or InGaAs (Indium Gallium Arsenic) detector is used. Some example images are showed in the below.

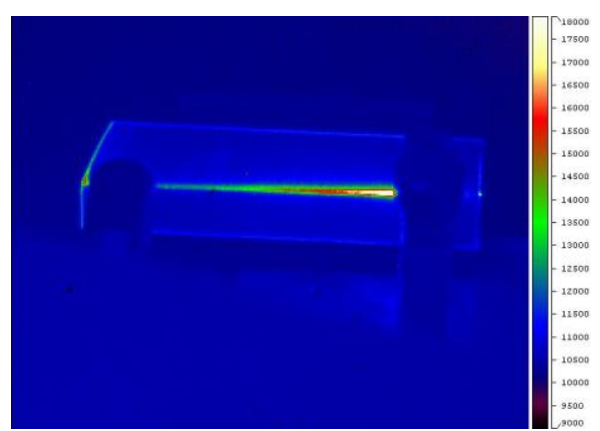

Fig. 1: waveguide profile

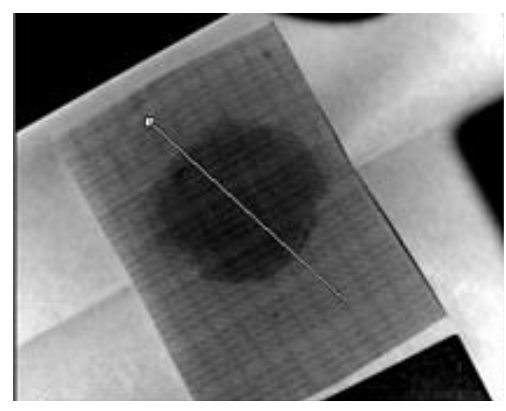

Fig. 2: water detection

In the market of optical communication, they use NIR ray of 1.3 um or 1.55 um in the optical fiber glass. Figure 1 shows the waveguide profile of LASER beam by using NIR camera. At this testing, beam intensity distribution is identified on the fiber glass.

Water has the absorbance of $1.45 \mathrm{um}$ area. Figure 2 is the image which shows water quantity on some cloth by seeing the reflection of 1.45 um radiation. 


\section{Merit - NIR Thermography}

For temperature measurement application, NIR are has some merit as thermography against MWIR or LWIR. We will introduce those as follows.

\subsection{Imaging through glass}

For example, in the application of LASER welding, glass or acrylic resin is used sometimes between LASER and human for protection from the high temperature particle. Or in the application of vacuum chamber, some glass is used for seeing the material inside the chamber. In these case, MWIR or LWIR thermography cannot see the target through glass or acrylic resin. We thermography manufacturer always say the customers to use sapphire or germanium window. However, sapphire or germanium is not so cheap, and not so good for transparency. Eventually it affects the image quality and the temperature measurement accuracy worse. In the area of NIR, glass or acrylic resin has the good transmittance. $\mathrm{NIR}$ thermography is suitable for temperature measurement through glass or acrylic resin. Example images are shown as follows. NIR camera is FLIR A6261 InGaAs detector of 0.9-1.7um sensitive, LWIR is FLIR A655sc Microbolometer detector of 7-14um sensitive.

- $\quad$ To see the building through office window

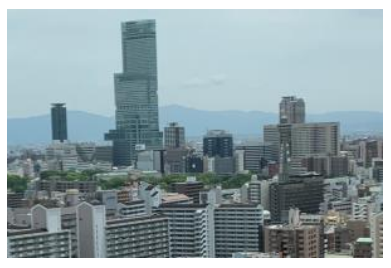

Fig. 3: Visible image

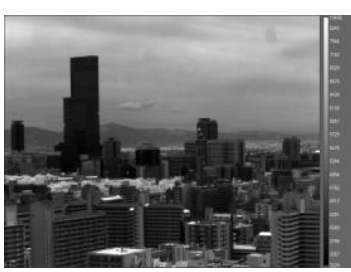

Fig. 4: NIR image

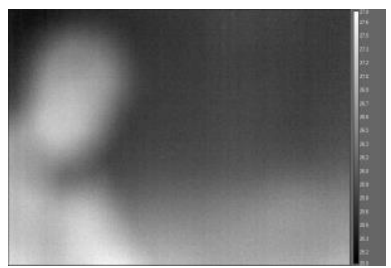

Fig. 5: LWIR image

My office is in the $19^{\text {th }}$ floor and there is big glass window of approximately $1 \mathrm{~cm}$ thickness. Each visible, NIR and LWIR image were captured. In visible and NIR image can see the building through glass, but not on LWIR. Some white part on LWIR image is the reflection of my body shape on the glass.

- $\quad$ To see the filament in the light bulb

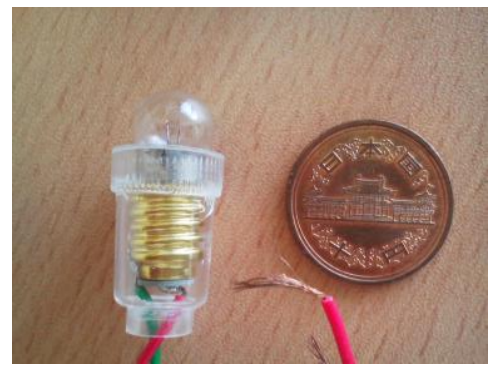

Fig. 6: Light bulb

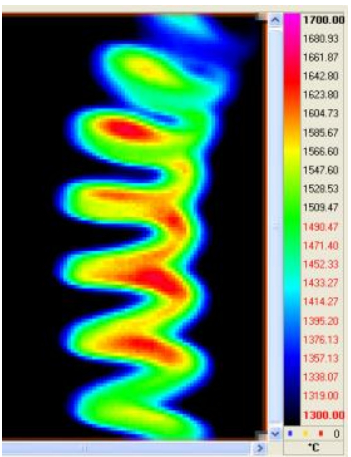

Fig. 7: IR image of filament

The temperature of the filament in the light bulb can be measured through glass. In the above thermography image, macro lens is used, then pixel resolution is $3.75 \mathrm{um} /$ pixel.

Like these images, NIR thermography can be used for temperature measurement through glass which is for protection, for vacuum chamber window, etc. 


\subsection{Emissivity effect}

As so called, we have to consider emissivity to use thermography. However, emissivity is not so easy to measure in the actual experimental situation. Because the material is not so clean or there is some contamination on the surface. On the metal, there are some oxide material. Or emissivity changes when the material temperature changes. Thermography or its software has the function of emissivity correction, but apparent temperature changes larger when the emissivity value is lower.

Now we consider Planck-law.

$$
I(\lambda, T)=\frac{2 h c^{2}}{\lambda^{5}} \cdot \frac{1}{e^{\frac{h c}{\lambda k T}-1}}
$$

Where $\lambda$ is wavelength, $T$ is absolute temperature, $h$ is Planck constant, $c$ is speed of light, $k$ is Boltzmann constant, and $l$ is spectral radiance. This equation shows that the temperature change $\Delta T$ brings the radiance change $\Delta$ $I$ at the constant wavelength. When the wavelength is longer, $\Delta T$ brings the bigger $\Delta I$, and it means that $\Delta I$ brings the bigger $\Delta T$. We can say that emissivity is equal to radiance change, so it brings more bigger temperature change when the wavelength is longer. And wavelength is shorter, temperature change is smaller under emissivity situation. Example images are shown as follows.

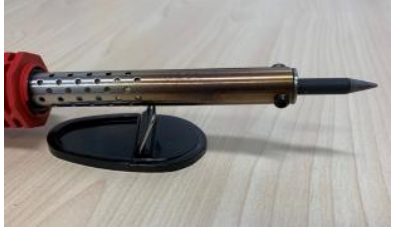

Fig. 8: Visible image

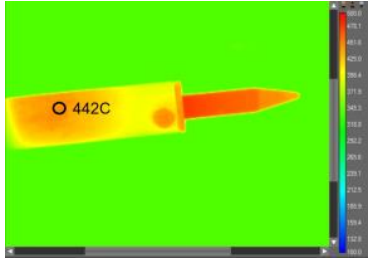

Fig. 9: NIR image

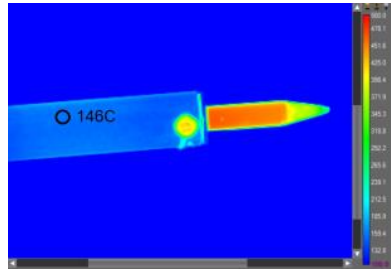

Fig. 10: LWIR image

These are soldering iron images. On the heater area of the soldering iron, Stainless is used as the material. Emissivity is low like $0.3-0.4$. On NIR and LWIR images, there is no emissivity correction settled. NIR image shows that the temperature of the heater area is $442 \mathrm{C}$ and almost same as the tip temperature of higher emissivity. Then, LWIR shows 146C of the heater area. This represents that NIR thermography does not affect the lower emissivity than longer wavelength thermography.

For further investigation, we tested other temperatures on NIR. Table 1 shows the temperature difference results between emissivity high (tip area) and low (heater area).

\begin{tabular}{|l|r|r|r|r|r|}
\hline Emissivity & \multicolumn{5}{|c|}{ Temperature showing } \\
\hline High (tip) & $600 \mathrm{C}$ & $550 \mathrm{C}$ & $500 \mathrm{C}$ & $450 \mathrm{C}$ & $400 \mathrm{C}$ \\
\hline Low (heater) & $554 \mathrm{C}$ & $507 \mathrm{C}$ & $453 \mathrm{C}$ & $406 \mathrm{C}$ & $360 \mathrm{C}$ \\
\hline
\end{tabular}

Table 1: Temperature difference

\subsection{Spatial resolution}

In the optical system, spatial resolution is given by Rayleigh criterion.

$$
d=1.22 \lambda \frac{f}{D}
$$

Where $\lambda$ is wavelength, $f$ is focal length, $D$ is aperture diameter and $d$ is the distance between two objects. When the wavelength is longer, spatial resolution is bigger. Thermography camera also has the definition of IFOV (Instantaneous Field Of View), but image quality and edge accuracy is different from wave length. Example images are shown as follows. 


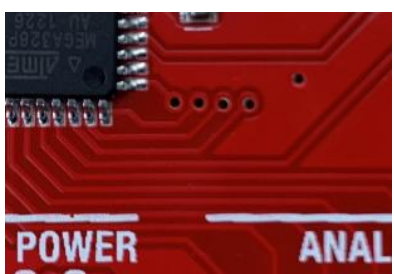

Fig. 11: Visible image

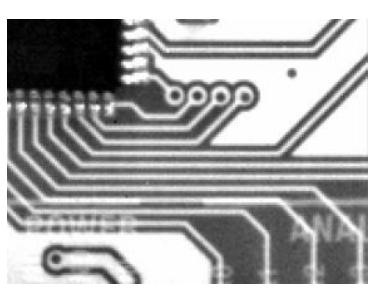

Fig. 12: NIR image

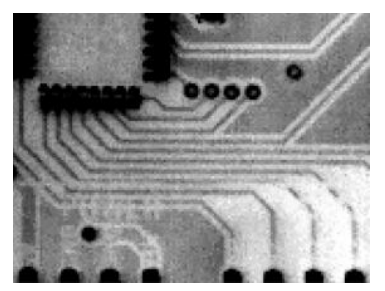

Fig. 13: LWIR image

The target is printed circuit board. NIR image is sharper than LWIR image especially on the edge. It means not only image quality is better, but also the temperature accuracy on the edge is better on NIR thermography.

\subsection{Lens variation}

MWIR and LWIR thermography lens material is germanium or silicon, so it is not common on the market. And the price is higher. In case of NIR thermography, optics is very common and much cheaper because lens material is glass. CCTV C-mount lens is available for NIR thermography.

\section{Limitation - NIR Thermography}

NIR thermography has some limitation or noticed things to use it by some other physics reasons.

\subsection{Need high temperature}

In the area of NIR, radiation is very low at the room temperature like $20 \mathrm{C}$ or $30 \mathrm{C}$. FLIR Systems is offering NIR thermography over $400 \mathrm{C}$ calibration. When the integration time is set longer, camera detector can get the smaller radiation. This means that NIR thermography can measure the temperature lower than 400C. But in this case, we should consider the noise from the environmental.

Table 2 shows the temperature range example.

\begin{tabular}{|l|}
\hline Temperature range \\
\hline $400-1200 \mathrm{C}$ \\
\hline $600-1500 \mathrm{C}$ \\
\hline $700-2500 \mathrm{C}$ \\
\hline
\end{tabular}

Table 2 : NIR thermography : temperature range example

- Lower temperature testing in lab room

To set the longer integration time, I tried to capture the lower temperature black body. Camera is FLIR A6261 InGaAs detector of 0.9-1.7um sensitive, black body diameter is 1 inch $(25.4 \mathrm{~mm})$. Figure 14 shows $200 \mathrm{C}$ black body, it can be seen by the red circle. Figure 15 is $150 \mathrm{C}$ black body and Figure 16 is $130 \mathrm{C}$. We can see little bit the black body circle of $150 \mathrm{C}$, but $130 \mathrm{C}$ is almost disappeared by the background.

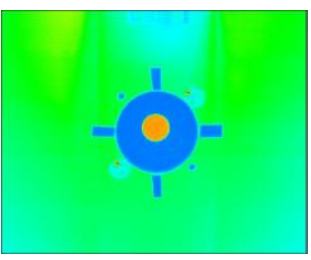

Fig. 14 : 200C black body

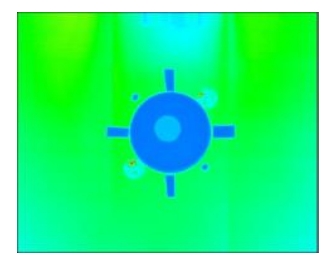

Fig. 15 : 150C black body

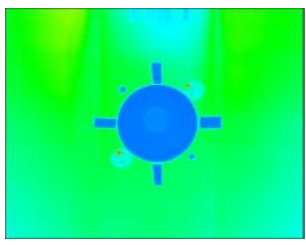

Fig. 16 : 130C black body 


\subsection{Noise from environmental}

Fluorescent light does not radiate NIR very much, but halogen or high intensity discharge lamp radiate NIR more. That will be noise to NIR thermography. Or Sunlight radiates NIR very much too. Using NIR thermography outside is not suitable.

- Curtain open / closed in the office room

As the example images, we captured the soldering iron images. One is the image which the curtain was closed on the window in my office, and the other is captured in the open curtain. Soldering iron temperature is around $250 \mathrm{C}$, so camera detector integration time is set longer. Fig.17 shows the image on the closed curtain and Fig.18 is the one on the open curtain.

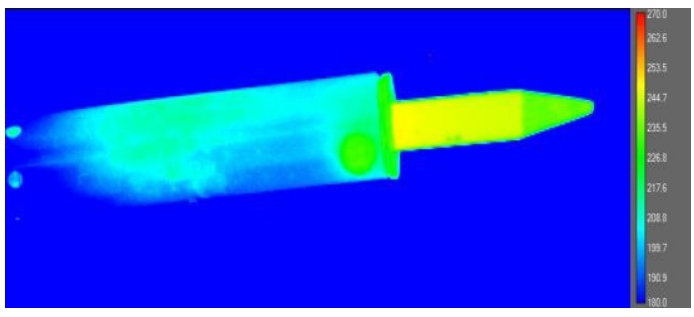

Fig. 17: 250C at curtain closed

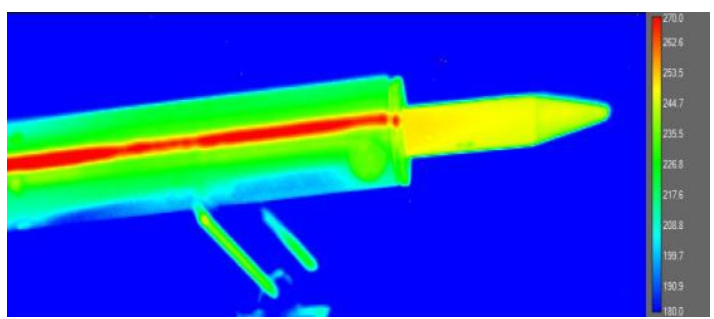

Fig. 18: 250C at curtain open

In Fig.18, some red line is appearing and the area around red line is become green, the color on tip area is not changed much though. It seems that the temperature is getting higher, but no. This is due to the reflection by sunlight from the room window. When the curtain is open, the sunlight is shining into the room. It effects the apparent temperature rising by the sunlight, even if the soldering iron temperature is not rising.

\section{- Lower temperature testing in lab room}

In the above experiment, $150 \mathrm{C}$ black body can be seen. Those images were taken in lab room, there is no window in the room, nor halogen light, high intensity discharge lamp. Then, can we say that it is possible to measure $150 \mathrm{C}$ temperature by NIR thermography? It is not easy to answer. There is some fluorescent light in the lab room. I captured $150 \mathrm{C}$ black body, when the fluorescent light is on and off. The following two images are the results.

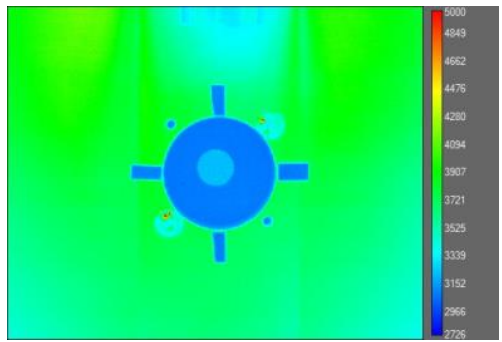

Fig. 19: 150C black body with fluorescent light on

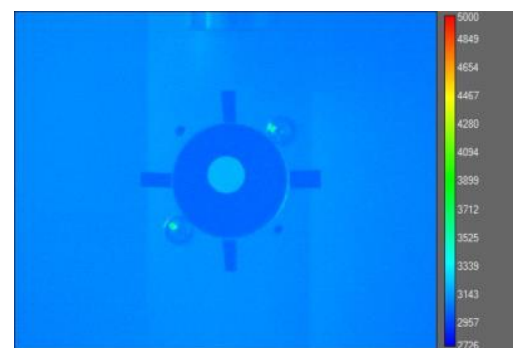

Fig. 20: 150C black body with fluorescent light off

Figure 19 image is brighter than Figure 20 image. This is because the fluorescent light is radiating into NIR camera by reflection. Even $150 \mathrm{C}$ black body area with fluorescent light on is little bit brighter than light off. Its difference is around 20 digital count under 3100 digital count of $150 \mathrm{C}$ black body. Black body emissivity is almost 1 and reflection should be almost zero. Still there is some difference on the black body area, it means that some scattering light in the room will be radiating into NIR camera.

Fluorescent light does not radiate NIR ray very much, but in this case of longer integration time, NIR thermography can detect the difference between fluorescent light on and off. It will affect the temperature measurement accuracy eventually. 
- Higher temperature testing in the office room

However, this effect from the environmental noise is lower when the target temperature is higher. Fig. 21, 22 shows each image of $600 \mathrm{C}$ soldering iron at curtain open, closed. This is because of shorter integration time, so the difference between curtain open and closed is not so much for the shorter integration time of NIR thermography.

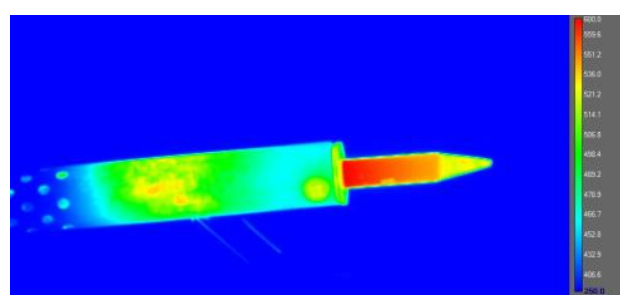

Fig. 21: 600C at curtain closed

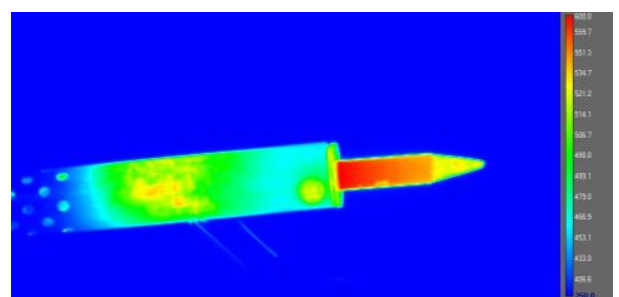

Fig. 22: 600C at curtain open

\section{Conclusion}

Thermography is using Mid-Wave Infrared (MWIR) or Long-Wave Infrared (LWIR) generally, however NearInfrared (NIR) can be used for thermography. It has some merit rather than MWIR, LWIR. In this paper, we introduced some merit. On the other hand, there are some limitation nor noticed things with NIR thermography. If the customers noticed these limitation, NIR thermography is very useful. We FLIR Systems will study NIR technology more and will offer the merit to the customers continuously. 